\title{
BDNF Modulates, But Does Not Mediate, Activity-Dependent Branching and Remodeling of Optic Axon Arbors In Vivo
}

\author{
Susana Cohen-Cory \\ Mental Retardation Research Center, Departments of Psychiatry and Neurobiology, University of California, Los Angeles, \\ Los Angeles, California 90095
}

The proper development of axon terminal arbors and their recognition of target neurons depend, in part, on neuronal activity. Neurotrophins are attractive candidate signals to participate in activity-dependent development and refinement of neuronal connectivity. In the visual system, brain-derived neurotrophic factor (BDNF) has been shown to modulate the elaboration and refinement of axonal arbors and to participate in the establishment of topographically ordered visual maps. By examining in vivo with time-lapse microscopy the effects of activity blockade and BDNF on optic axon arborization, I show that the dynamic mechanisms by which neurotrophins and neuronal activity regulate axon arborization differ. Acute retinal activity blockade by intraocular injection of tetrodotoxin (TTX) rapidly and significantly increased branch addition and elimination, thus interfering with axon branch stabilization. The effects of activity blockade on branch dynamics resulted in increased arbor complexity in the long term and were prevented

In most vertebrate species the establishment of precisely ordered neuronal connections involves a gradual process in which afferent axonal arbors initially branch widely over their target tissue and then gradually refine by withdrawing branches from topographically inappropriate areas (Antonini and Stryker, 1993a; Roskies et al., 1995; Katz and Shatz, 1996). This gradual remodeling of axonal arbors involves a dynamic process in which axonal branches are constantly added and eliminated, until stable synaptic connections are retained (Nakamura and O'Leary, 1989; O'Rourke and Fraser, 1990). In the visual system, patterns of neuronal activity modulate the development and refinement of visual connections. For example, in the absence of action potential activity, axons projecting to their target regions fail to segregate in retinotopic specific lamina and elaborate arbors that are more complex than those developing with normal visual input (Reh and Constantine-Paton, 1985; Sretavan et al., 1988; Kobayashi et al., 1990; Antonini and Stryker, 1993b). At least two mechanisms have been proposed to account for the formation of exuberant arbors in the absence of visual activity. One possibility is that the elimination of side branches or the pruning of axon

\footnotetext{
Received July 12, 1999; revised Aug. 20, 1999; accepted Aug. 27, 1999.
}

This work was supported by National Institutes of Health Grant EY11912 and by awards from the Alfred P. Sloan, Stein/Oppenheimer, University of California Los Angeles Frontiers of Science, and Beckman foundations. I thank J. Cueva for assistance with initial experiments, C. Colwell for help with electrophysiological recordings, T. Vu for technical assistance, and R. Frostig, C. Colwell, B. Lom, and J. Cogen for discussions and comments on this manuscript.

Correspondence should be addressed to Dr. Susana Cohen-Cory, Mental Retardation Research Center, 760 Westwood Plaza, NPI 78-148, University of California, Los Angeles, Los Angeles, CA 90095. E-mail: scohenco@ucla.edu.

Copyright (C) 1999 Society for Neuroscience 0270-6474/99/199996-08\$05.00/0 by altering endogenous BDNF levels at the target. BDNF promoted axon arborization by increasing branch addition and lengthening, without affecting branch elimination. Activity blockade, however, did not prevent the growth-promoting effects of BDNF, indicating that BDNF can affect axon arborization even in the absence of activity. Together this evidence indicates that BDNF acts as a modulator, but not as a direct mediator, of activity during the morphological development of neurons. Consequently, neuronal activity and BDNF use distinct but interactive mechanisms to control the development of neuronal connectivity; BDNF modulates axon arborization by promoting growth, neuronal activity participates in axon branch stabilization, and together these two signals converge to shape axon form.

Key words: BDNF; neuronal activity; TTX; retinal ganglion cells; axon branching; Xenopus laevis

terminal arbors that occurs during normal map refinement is inhibited or delayed in the absence of neuronal activity (Kobayashi et al., 1990; Shatz, 1996). Another possibility is that neural activity blockade promotes sprouting and growth of axonal terminal arbors by interfering with target recognition and synapse stabilization mechanisms (Cline, 1991; Antonini and Stryker, 1993b; Shatz, 1996). Although both mechanisms support a role for neuronal activity as a stabilizing force during competition for synaptic inputs, no direct evidence describes the mechanism by which action potential activity influences axon arbor morphology.

Experimental evidence suggests that competition between axonal inputs for common postsynaptic sites involves activitydependent competition for neurotrophic substances (Katz and Shatz, 1996; Snider and Lichtman, 1996). One mechanism that may explain such competition is that neuronal activity directly regulates the production and release of a trophic factor (Thoenen, 1995). The neurotrophins have been implicated as candidates to mediate activity-dependent development and refinement of synaptic connections. Increasing evidence suggests that neurotrophin synthesis and release depend on neuronal activity (Herzog et al., 1994; Lindholm et al., 1994; Thoenen, 1995; Blochl and Thoenen, 1996) and that neurotrophins can modulate neuronal activity (Lohof et al., 1993; Kang and Schuman, 1995; Thoenen, 1995; Prakash et al., 1996; Snider and Lichtman, 1996). Moreover, the development of topographically ordered visual maps in the cortex (Maffei et al., 1992; Cabelli et al., 1995) and the dynamic development of optic axon terminal arbors depend on endogenous neurotrophin function (CohenCory and Fraser, 1995). 
One requirement for a molecule to be directly involved in activity-dependent synaptic rearrangements is its ability to influence axon terminal arborization (Snider and Lichtman, 1996). Our previous work demonstrates that the neurotrophin brainderived neurotrophic factor (BDNF) modulates optic axon remodeling in vivo and therefore suggests that BDNF also acts as a signal to mediate activity-dependent synaptic rearrangement. The rapid ( $<2 \mathrm{hr}$ ) and significant effect that BDNF exerts on retinal ganglion cell (RGC) axon arborization and complexity in vivo (Cohen-Cory and Fraser, 1995), as well as the patterns of BDNF expression in the developing Xenopus visual system (Cohen-Cory and Fraser, 1994; Cohen-Cory et al., 1996), is consistent with a direct effect on optic axons and suggested the direct involvement of BDNF in the activity-dependent establishment of connections between RGCs and their targets. By using the Xenopus visual system as an in vivo model, I have now examined whether in Xenopus, as in mammals, retinal activity plays a significant role in the elaboration of RGC axonal arbors. By imaging the morphology of individual RGC axon arbors over time in tadpoles in which retinal action potential activity was blocked, I examined the mechanisms by which neuronal activity influences axon form. Moreover, I studied whether the effects of blocking action potential activity on RGC axon terminal arbor complexity are modifiable by BDNF. These studies indicate that acute neuronal activity blockade rapidly and significantly increases axon terminal arbor remodeling by increasing branch addition and elimination without significantly altering arbor length. This resulted in a significant increase in RGC axon arbor complexity in the long term. These results significantly differ from those obtained by perturbing endogenous BDNF at the target optic tectum and are further modified by tectal BDNF. Consequently, neuronal activity and BDNF use distinct but interactive mechanisms to modulate axon terminal arbor complexity in vivo; neuronal activity modulates axon branch stabilization, and BDNF modulates axon growth, and together these two signals converge to shape axon form.

\section{MATERIALS AND METHODS}

Xenopus laevis tadpoles were obtained from in vitro fertilization of oocytes obtained from adult females primed by injection of human chorionic gonadotropin. Tadpoles were reared in a modified rearing solution [60 mM NaCl, $0.67 \mathrm{~mm} \mathrm{KCl}, 0.34 \mathrm{~mm} \mathrm{Ca}\left(\mathrm{NO}_{3}\right)_{2}, 0.83 \mathrm{~mm}$ $\mathrm{MgSO}_{4}, 10 \mathrm{~mm}$ HEPES, pH 7.4, and $40 \mathrm{mg} / 1$ gentamycin] plus $0.001 \%$ phenylthiocarbamide to prevent melanocyte pigmentation. Staging was according to the method of Nieuwkoop and Faber (1956). Animal procedures were approved by the University of California Los Angeles Office for Protection of Research Subjects, Animal Research Committee.

Electrophysiological recordings. Laminar field potentials in response to visual stimulation were electrophysiologically recorded from stage 43-45 anesthetized tadpoles to test the effectiveness of the tetrodotoxin (TTX) treatment to eliminate all retinal action potential activity. Tadpoles were anesthetized by immersion in MS-222 in modified rearing solution and placed over an agar cushion. Evoked field potentials were recorded from the tectum using glass micropipettes filled with Ringer's solution (resistance, 20-30 M $\Omega$ ). A monopolar recording paradigm was used, with the electrode placed in the tectum and a reference electrode placed in the solution-filled recording dish. Both ON- and OFF-light responses after flash stimulation were readily recorded (50-100 $\mu \mathrm{V} ; 100$ msec delay; data not shown) in the developing optic tectum of intact tadpoles $(n=8$ out of 8 tadpoles, each recorded at multiple sites within the tectal neuropil). No light-evoked responses, however, could be recorded in tadpoles after intraocular injection of $4 \mathrm{nl}$ of $100 \mu \mathrm{m}$ TTX (the concentration used in these studies), even $6-8 \mathrm{hr}$ after injection $(n=5$ tadpoles; data not shown). In only one out of the five tadpoles could a weak light-evoked response (magnitude 10 times lower than those recorded from control tadpoles) be recorded in 1 out of every 10 trials $8 \mathrm{hr}$ after TTX injection. When lower TTX concentrations were injected, tadpoles recovered more readily from the TTX treatment (6-10 $\mathrm{hr}$ after injection; swimming and twitch behavior) and showed light-evoked responses of the same magnitude as those of controls.

In vivo microscopy imaging and drug treatment. Stage 43 tadpoles were anesthetized, and individual RGCs and their axons were labeled by microinjection of minute amounts of the lipophilic vital dye 1,1'dioctadecyl-3,3,3',3'-tetramethylindocarbocyanine perchlorate [DiI; DiIC18(3); Molecular Probes, Eugene, OR] into the nasomedial quadrant of the retina. Tadpoles were screened $18 \mathrm{hr}$ later for the presence of DiI-labeled retinal arbors in the tectum, and those tadpoles with one to two clearly distinguishable DiI-labeled arbors, containing at least one or two branches, were selected and used for experimentation. Tadpoles were anesthetized and imaged with a Bio-Rad (Hercules, CA) laserscanning confocal microscope. Individual optical sections were recorded at $2 \mu \mathrm{m}$ intervals through the full extent of the arbor within the optic tectum. After the first imaging session, either $4 \mathrm{nl}$ of a $100 \mu \mathrm{m}$ TTX solution (Research Biochemicals, Natick, MA ) or control vehicle solution (10\% NiuTwitty) was microinjected directly into the eye of the developing tadpoles with care to minimize mechanical damage. Immediately after the TTX injection, $0.2-1.0 \mathrm{nl}$ of vehicle solution, $200 \mathrm{ng} / \mu \mathrm{l}$ recombinant human BDNF (a gift of Amgen, Thousand Oaks, CA), specific function-blocking antibodies to BDNF $(200 \mu \mathrm{g} / \mathrm{ml}$; a gift of J. Carnahan) (Ghosh et al., 1994), or control IgG (200 $\mu \mathrm{g} / \mathrm{ml}$ nonimmune rabbit $\mathrm{IgG}$ ) in vehicle solution was pressure injected with a fine-pulled glass micropipette directly into the ventricle and subpial space overlying the caudalmost tectum (Cohen-Cory and Fraser, 1995). Tadpoles were kept in fresh rearing solution, in the dark, after treatment and between observations. The rates and dynamics of RGC axonal branching were followed by confocal microscopy immediately after injection, and every 2 $\mathrm{hr}$ after the initial observation, for a total of $6 \mathrm{hr}$ and again at $24 \mathrm{hr}$.

Data analysis. The effects of each treatment on arbor dynamics were examined by analyzing individual confocal optical sections through the entire extent of the arbor at each observation time point with the aid of the Metamorph software (Universal Imaging Corporation, West Chester, PA). Several morphological parameters were measured. Extensions from the main axon were classified as branches (longer than $5 \mu \mathrm{m}$ ) or spikes (shorter than $5 \mu \mathrm{m}$ ). The number of individual branches or spikes, gained or lost, and the number of preexisting branches that change their length by at least $20 \mu \mathrm{m}$ from one observation interval to the next were scored regardless of their length change. In addition, the number of total branches per observation time point was determined. For the analysis of arbor complexity at $24 \mathrm{hr}$, total axon length was determined by measuring the difference in total length of the main stem and proximal and distal branches at 24 versus $0 \mathrm{hr}$, and the difference in total branch number was determined by counting the total number of branches (proximal and distal branches) at 24 versus $0 \mathrm{hr}$. Axon arbor reconstructions were obtained by digitally tracing individual arbors from individual optical planes with the Metamorph software. Repeated measures ANOVA (SYSTAT; SPSS) and post hoc Tukey tests were used for the statistical analysis of data. Significance was $p \leq 0.05$.

\section{RESULTS}

The effect of silencing retinal activity on the dynamics of RGC axon arborization was studied in live stage 45 Xenopus laevis tadpoles in which action potential activity was inhibited by intraocular injection of the sodium channel blocker TTX. Minute amounts of the lipophilic dye DiI were injected into tadpole retinae to visualize individual RGC axon arbors in the optic tectum. The morphology of individual DiI-labeled RGC axon arbors was followed by time-lapse in vivo confocal microscopy. Immediately after the initial microscopic examination of axon arbor morphology, $4 \mathrm{nl}$ of $100 \mu \mathrm{m}$ TTX was pressure injected into the eye of each anesthetized tadpole. This amount of TTX effectively blocked all retinal action potential activity (see Materials and Methods). Analysis of individual axon arbor morphology at $2 \mathrm{hr}$ intervals for at least $6 \mathrm{hr}$ revealed active remodeling of axon arbors both in TTX- and control-treated tadpoles (Figs. 1, 2). Axon arbor dynamics was evaluated by measuring the number of branches that are added or eliminated in each $2 \mathrm{hr}$ time point and the number of preexisting branches that lengthened or shortened. In addition, the number of spikes (dynamic processes $<5$ 


\section{Control}
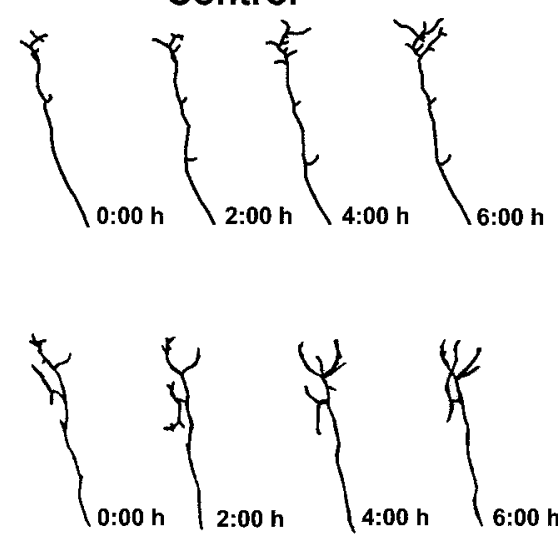
namics of RGC axon arborization are illustrated by reconstructions of sample arbors followed over time after intraocular injection of TTX (top right) or control (top left) solution. For comparison, tracings of a sample arbor in a tadpole with intraocular TTX and tectal BDNF injections (bottom) are also presented (see text and also Fig. 3). Posterior is up, and medial is to the left.

Figure 1. Action potential activity blockade increases the remodeling of RGC axonal arbors in vivo. The effects

$\mu \mathrm{m})$ added or eliminated in every $2 \mathrm{hr}$ time period was evaluated. Blocking retinal action potential activity significantly altered the number of branches added and eliminated at every observation time point. On average, RGC axons in TTX-treated tadpoles added $2.3 \pm 0.23$ new branches every $2 \mathrm{hr}$, whereas RGC axons in control-treated tadpoles added $1.3 \pm 0.14$ new branches (Fig. $2 A$ ). These effects were observed at the first $2 \mathrm{hr}$ time point and persisted throughout the entire imaging period (data not shown). Similarly, TTX significantly increased the number of branches eliminated per RGC axon, compared with RGCs in controltreated tadpoles $(\mathrm{TTX}=1.22 \pm 0.14$ vs control $=0.66 \pm 0.08$ branches eliminated every 2 hr; Fig. $2 B$ ). TTX treatment, however, did not affect the lengthening or shortening of preexisting branches (Fig. $2 A, B$ ) or the addition or elimination of spikes (Fig. $2 A, B)$. Thus, the increases in branch addition and elimination observed after intraocular TTX injection indicate that RGC axonal arbors are more dynamic in the absence of action potential activity. Moreover, these results indicate that normal patterns of neuronal activity are required for the stabilization of new branches but do not influence the rate at which existing branches grow.

We demonstrated previously that BDNF modulates axon arborization by specifically promoting growth (Cohen-Cory and Fraser, 1995). That is, increasing tectal BDNF levels significantly increased the addition of new branches and spikes as well as the lengthening of preexisting branches without affecting branch and spike elimination or branch shortening (see also Fig. 3). Thus, although both intraocular TTX injections and perturbations in BDNF tectal levels alter axon branch dynamics, not all parameters of axon arbor dynamics are affected in the same manner by these two treatments. Silencing activity with TTX significantly increased branch addition and elimination without affecting branch length and spike number, whereas increasing BDNF tectal levels increased all parameters that reflect axon growth (see Cohen-Cory and Fraser, 1995) (see also Fig. 3). This suggests that BDNF modulation of RGC axon arborization might be independent of activity. To address this issue directly, I followed RGC
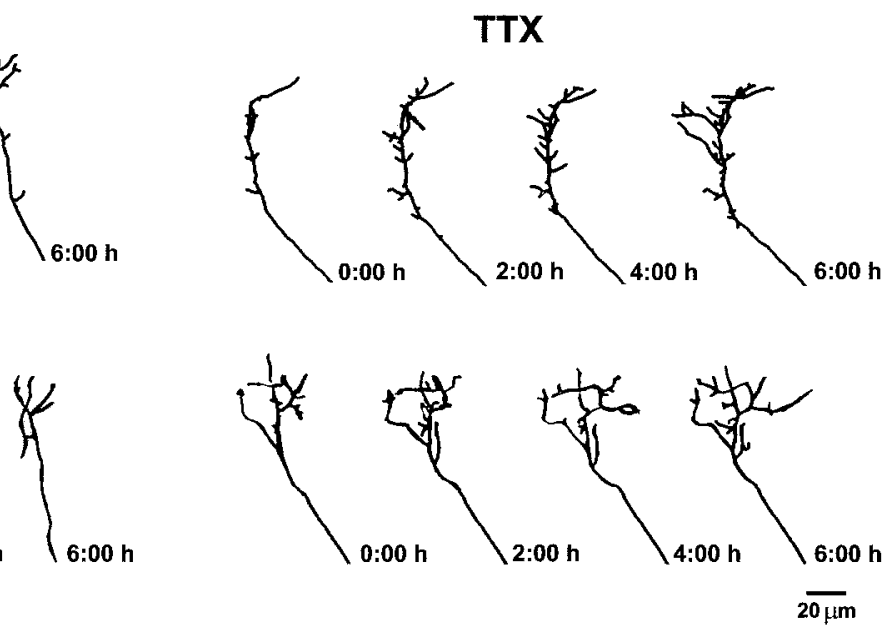

\section{TTX + BDNF}
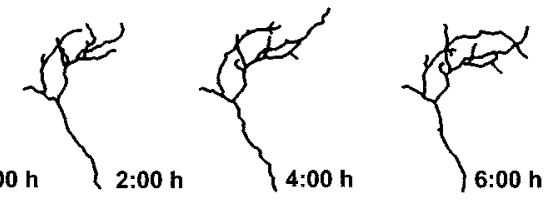

axon arborization dynamics in tadpoles that were treated with both TTX to silence activity and BDNF to increase tectal BDNF levels or with function-blocking BDNF antibodies to neutralize endogenous tectal BDNF. As internal controls for these experiments, individual DiI-labeled RGC axons were followed in tadpoles in which tectal BDNF levels were altered by injection of BDNF or anti-BDNF but the TTX treatment was omitted (Fig. 3).

Combining intraocular TTX injections with perturbations in BDNF tectal levels resulted in RGC axonal arbors with branch dynamics that significantly differed from those in axons from tadpoles treated with TTX, BDNF, or anti-BDNF alone. In agreement with previous results, increasing the endogenous levels of BDNF by tectal injection of BDNF alone significantly increased the number of branches added, whereas anti-BDNF significantly decreased branch addition (control $=1.30 \pm 0.15$; $\mathrm{BDNF}=2.22 \pm 0.24$; anti-BDNF $=0.75 \pm 0.15$; Fig. $3 A)($ see also Cohen-Cory and Fraser, 1995). Surprisingly, the number of branches added per RGC axon after TTX + BDNF treatment did not differ from that of RGC axons in control-treated tadpoles $($ control $=1.30 \pm 0.15 ;$ TTX + BDNF $=1.53 \pm 0.17$; Fig. $3 A)$. Similarly, the combined treatment of TTX and function-blocking antibodies to BDNF did not alter the number of branches added per $\mathrm{RGC}$ (control $=1.30 \pm 0.15$; $\mathrm{TTX}+$ anti-BDNF $=1.22 \pm$ 0.18; Fig. $3 A$ ). Thus perturbations in either tectal BDNF levels or activity blockade with TTX independently caused significant changes in the number of new branches added, but when these two treatments were combined no apparent effect on branch addition was observed. These effects were consistent over the $6 \mathrm{hr}$ time course (data not shown; for qualitative measure see Fig. 1). Perturbations in tectal BDNF levels performed simultaneously with TTX treatment affected other axon branch parameters in a different manner. In those parameters in which TTX had no independent effect but BDNF significantly affected axon branch dynamics, the effects of the combined treatment with TTX and $\mathrm{BDNF}$ resembled those of BDNF alone. For example, the number of branches that lengthened in each $2 \mathrm{hr}$ interval was signif- 

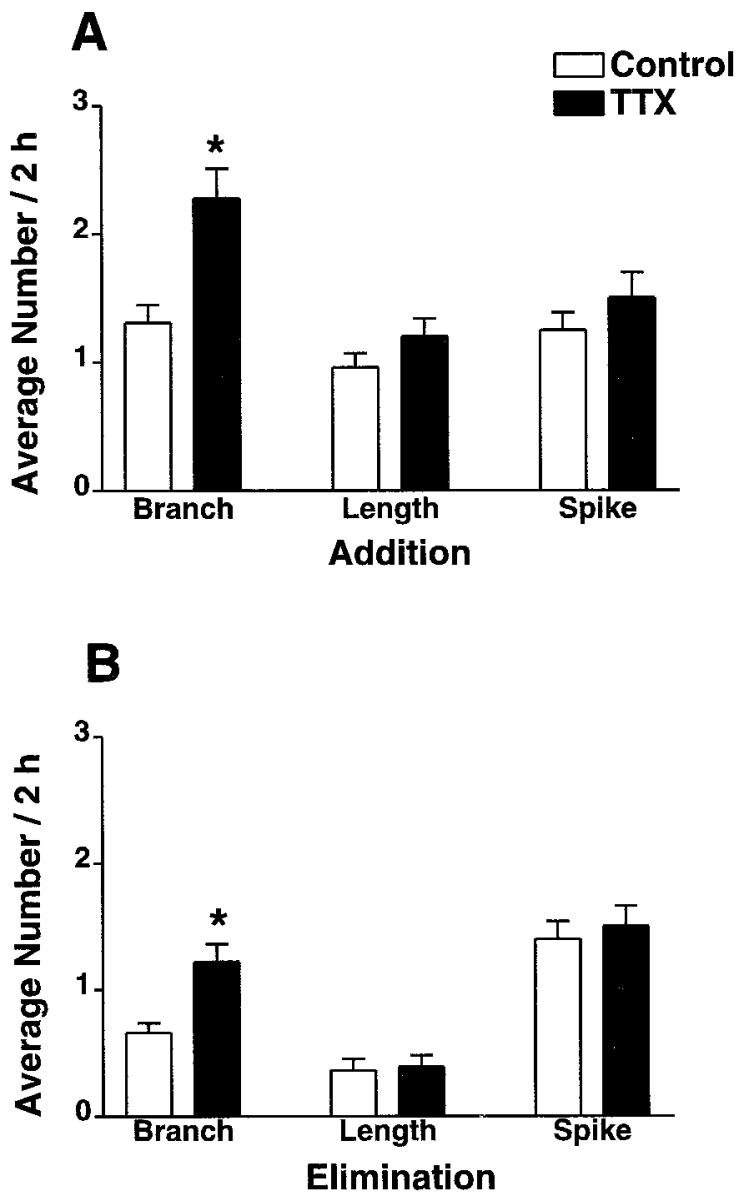

Figure 2. Action potential activity blockade increases addition and elimination of RGC axonal branches. To quantify the effects of activity blockade on RGC axon arbor dynamics, three morphological parameters were evaluated: addition $(A)$ and elimination $(B)$ of individual branches $(>5 \mu \mathrm{m})$, changes in the length of preexisting branches [lengthening $(A)$; shortening $(B)$ ], and addition $(A)$ and elimination $(B)$ of individual spikes $(<5 \mu \mathrm{m})$ are expressed as the average number of changes per $2 \mathrm{hr}$ time interval in arbors followed for $6 \mathrm{hr}(n=20$ arbors per condition). For each parameter and condition the mean \pm SEM is shown. Statistical analysis was by repeated measures ANOVA. * indicates significantly different from control, $p \leq 0.02$.

icantly increased by the combined treatment with TTX and BDNF to values similar to those resulting from treatment with $\mathrm{BDNF}$ alone $($ control $=0.93 \pm 0.11 ; \mathrm{TTX}=1.3 \pm 0.14 ; \mathrm{BDNF}=$ $1.74 \pm 0.22$; TTX + BDNF $=1.80 \pm 0.17$; see Fig. $3 B$ ). Similarly, combining TTX and BDNF treatments significantly increased the number of spikes added to values similar to those observed in RGC axons of tadpoles treated with BDNF alone (control $=1.25$ $\pm 0.14 ; \mathrm{TTX}=1.5 \pm 0.2 ; \mathrm{BDNF}=2.44 \pm 0.33 ; \mathrm{TTX}+$ $\mathrm{BDNF}=2.11 \times 0.24$; graph not shown). Neutralizing endogenous tectal BDNF with function-blocking BDNF antibodies elicited a small but significant increase in the number of branches shortened every $2 \mathrm{hr}$ (control $=0.36 \pm 0.09$; anti-BDNF $=0.81$ $\pm 0.13)$, an effect that was not detected as significant in our previous study (Cohen-Cory and Fraser, 1995). The differential effects that BDNF and anti-BDNF elicit on branch lengthening and branch shortening (see Fig. 3B,D) may be a consequence of differential threshold responses to BDNF signaling by previously established branches (see also Song et al., 1997). Similar to the effect of BDNF on branch lengthening, the effect of anti-BDNF on branch shortening was not influenced by intraocular TTX injection (control $=0.36 \pm 0.09$; $\mathrm{TTX}=0.33 \pm 0.1$; anti$\mathrm{BDNF}=0.81 \pm 0.13$; $\mathrm{TTX}+$ anti-BDNF $=0.75 \pm 0.12$; see Fig. $3 D)$. Consequently, blocking action potential activity with TTX did not alter the effects of exogenous and endogenous BDNF on RGC axon branch elongation or spike formation. In contrast, the effects of TTX could be blocked by perturbations in tectal BDNF levels. TTX significantly increased branch elimination, whereas the number of branches eliminated in BDNF- or anti-BDNFtreated tadpoles did not differ from that of controls (control = $0.66 \pm 0.08$; $\mathrm{TTX}=1.23 \pm 0.14 ; \mathrm{BDNF}=0.85 \pm 0.14$; anti$\mathrm{BDNF}=0.75 \pm 0.16$; Fig. $3 C$ ). In tadpoles treated with both TTX and exogenous BDNF, branch elimination did not differ from that of controls (TTX $+\mathrm{BDNF}=0.75 \pm 0.12$; see above and Fig. $3 C$ ). However, in TTX + anti-BDNF-treated tadpoles axon branch elimination significantly increased versus control, to a value similar to that observed after treatment with TTX alone $(\mathrm{TTX}+$ anti-BDNF $=1.4 \pm 0.18$; see above and Fig. $3 C)$. Together, these results indicate that exogenous BDNF can prevent most of the effects that result from blocking action potential activity with TTX and suggest that endogenous BDNF and neuronal activity act via distinct mechanisms to modulate RGC axon terminal arborization in vivo.

Comparing RGC axon morphology before and $24 \mathrm{hr}$ after treatment provided a cumulative measure of the effects of neural activity blockade and BDNF on axon terminal arbor complexity. Tracings of sample arbors illustrate the effects of each treatment, as well as the range of morphologies and responses to individual treatments (Fig. 4). In addition, distribution plots of the difference in branch number $24 \mathrm{hr}$ after treatment show the means and variability of the responses of individual arbors to all treatments (Fig. 5). The difference in total branch number and in total axon arbor length for each individual arbor between 0 and $24 \mathrm{hr}$ provides a more quantitative measure of the effects of individual treatments in the complexity of RGC axon terminal arbors (Fig. 6). RGC axons from tadpoles in which neural activity was blocked by intraocular TTX injection were significantly more complex at $24 \mathrm{hr}$ than RGC axons developing in normal tadpoles, as shown by the significantly increased change in branch number in the TTX-treated tadpoles compared with controls (Fig. 6; see also Fig. 4). These effects of TTX on axon arbor complexity differ from the very significant effects of BDNF. RGC axons in tadpoles exposed to increased BDNF tectal levels had significantly more branches and were significantly longer than RGC axons in control-treated tadpoles. Although BDNF and TTX independently increased axon arbor complexity, the overall change in complexity of RGC axon terminal arbors in TTX + BDNFtreated tadpoles was similar to that of RGC axons in control tadpoles (Fig. 6; see also Fig. 4). In contrast, RGC axons in tadpoles treated with antibodies to BDNF alone or with antibodies to BDNF in combination with TTX were simpler than controls $24 \mathrm{hr}$ after treatment as exemplified by the significantly lower increase in branch number versus controls (Fig. 6; see also Fig. 4). Therefore, under all conditions, the complexity of RGC axons at $6 \mathrm{hr}$ (data not shown) and at $24 \mathrm{hr}$ (Fig. 6) is in accordance with what would be expected on the basis of the summation of all effects that each individual treatment elicited on all parameters of axon branch dynamics in axons followed every $2 \mathrm{hr}$.

\section{DISCUSSION}

An important and long-standing question in development neurobiology is the extent to which neuronal activity participates in the 

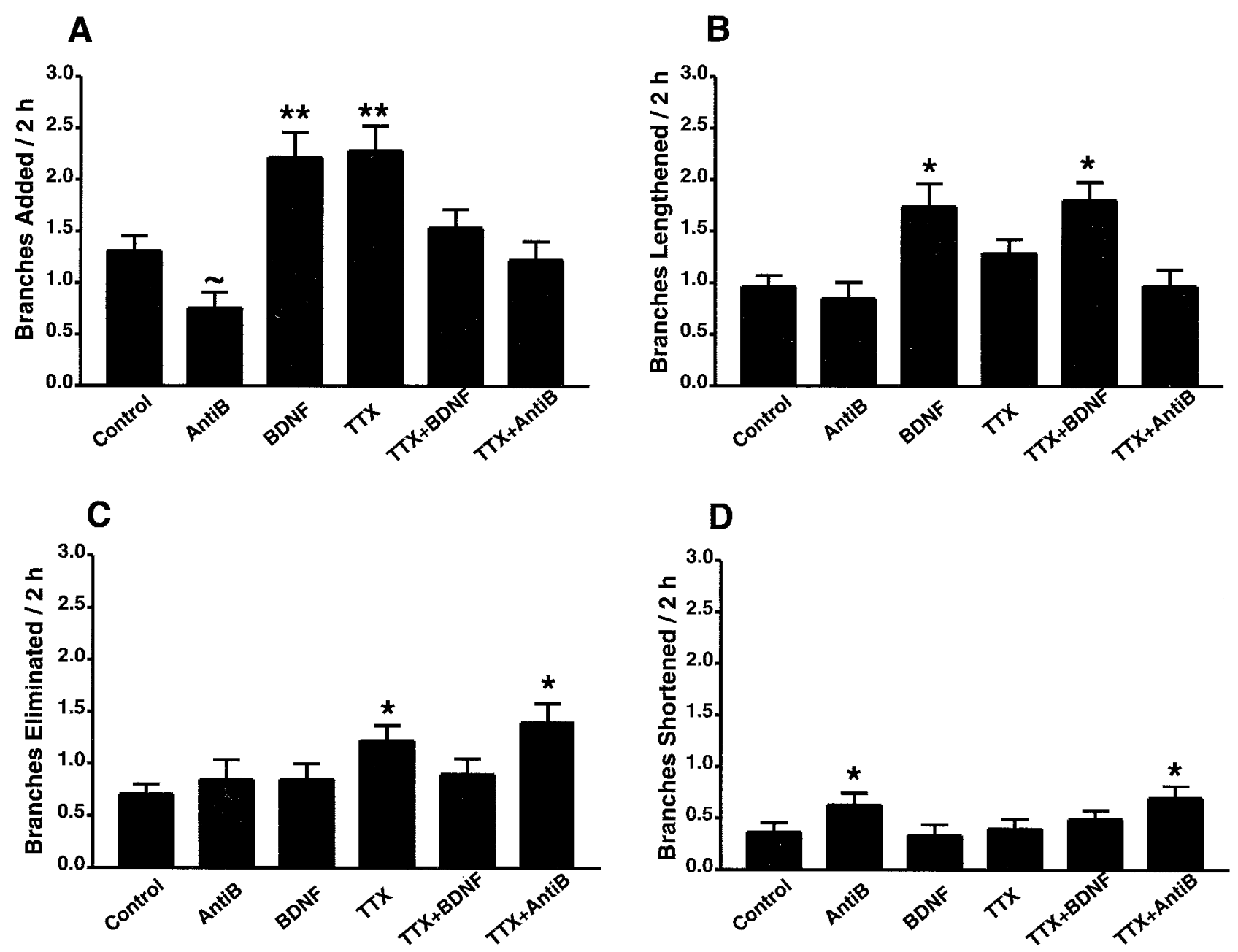

Figure 3. Action potential activity and BDNF differentially modulate the dynamics of RGC axon arborization in vivo. Comparing TTX effects on axon branch dynamics with those elicited by alterations in tectal BDNF levels illustrates the differential response of RGC axons to each treatment. In addition, the results of combining intraocular TTX injections with tectal injections of BDNF or anti-BDNF (AntiB) show that TTX influence on arbor dynamics is modified by BDNF. The effects of each treatment on axon branch dynamics are illustrated by quantifying the number of branches added $(A)$, or eliminated $(C)$, and the number of preexisting branches that lengthened $(B)$, or shortened $(D)$, for each treatment. These values are expressed as the average number of changes per $2 \mathrm{hr}$ time interval in arbors followed for $6 \mathrm{hr}(n=20$ arbors per condition for control, TTX, and TTX $+\mathrm{BDNF} ; n=$ 13 for BDNF; and $n=17$ for anti-BDNF and TTX + anti-BDNF). For each parameter and condition the mean \pm SEM is shown. Repeated measures ANOVA was used for the statistical analysis of data. ** indicates significantly different from all other groups except BDNF versus TTX, $p \leq 0.01$; $*$ indicates significantly different from control, $p \leq 0.05$; and $\sim$ indicates significantly different from control by unpaired $t$ test, $p \leq 0.02$.

development of neuronal connectivity (Constantine-Paton et al., 1990; Katz and Shatz, 1996; Snider and Lichtman, 1996). Neuronal activity may act directly to modulate neuronal connectivity or indirectly by enabling other developmental cues, such as neurotrophic factors, to guide the formation of appropriate connections (Katz and Shatz, 1996; Crair, 1999). By examining in vivo the events that lead to the formation of RGC axon terminal arbors, I now provide evidence that neuronal activity and neurotrophins act via distinct but interactive mechanisms to modulate axon arborization. A detailed analysis of morphological parameters from time-lapse-imaged RGC axonal arbors in live Xenopus tadpoles provides direct evidence that in the absence of neuronal activity, RGC axon terminal arbors significantly increase their complexity by actively extending and retracting axonal branches. Thus, in young developing tadpoles, as in mammals (Sretavan et al., 1988), neuronal activity plays an important role in the elaboration of RGC axonal arbors. Most studies investigating the role of neuronal activity during axon arborization have used pharma- cological agents such as TTX to block action potential activity chronically in the developing brain but only later examined the resulting axon arbor morphologies at a single time point (Meyer, 1983; Reh and Constantine-Paton, 1985; Sretavan et al., 1988; Kobayashi et al., 1990; Antonini and Stryker, 1993b). The mistargeting errors and increased axonal branching observed after chronic activity blockade suggested multiple mechanisms that may account for the effects of interfering with activity signaling. Potential mechanisms include interference with target recognition (Catalano and Shatz, 1998; Dantzker and Callaway, 1998), interference with arbor pruning (Kobayashi et a., 1990; Shatz, 1996), and/or failure to stabilize synaptic connections (ConstantinePaton et al., 1990; Cline, 1991; Antonini and Stryker, 1993b; Shatz, 1996). By acutely blocking action potentials via intraocular TTX injection in developing tadpoles and examining axon branch dynamics over time, I directly demonstrate that silencing retinal activity very rapidly affects axon branch stabilization. Within $2 \mathrm{hr}$ of TTX treatment, a significant increase in axon branch remod- 

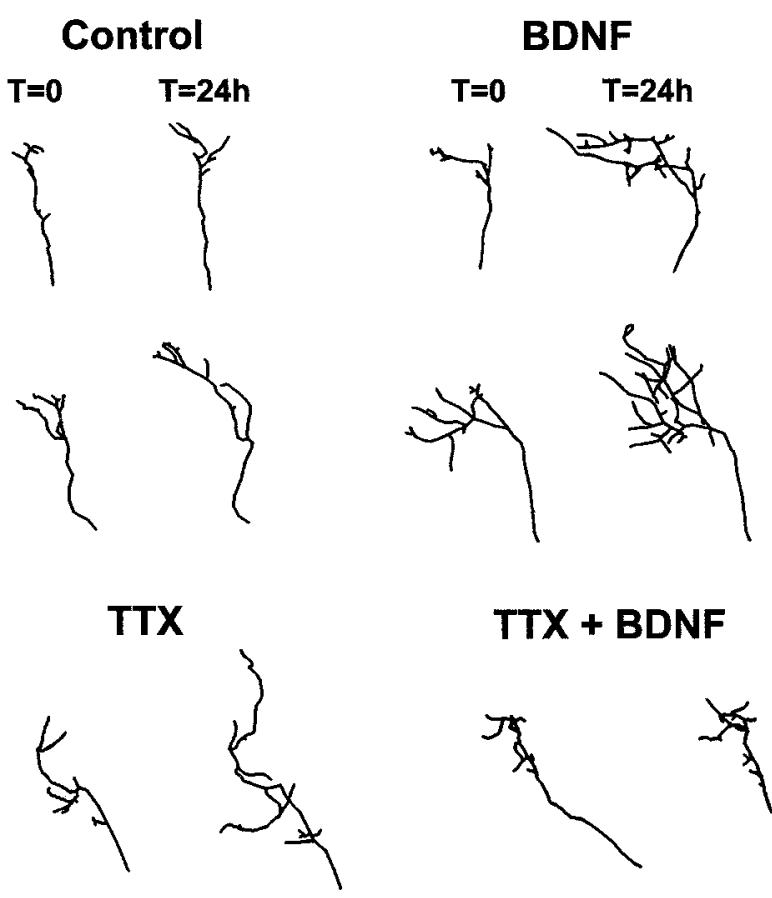

\section{TTX + BDNF}
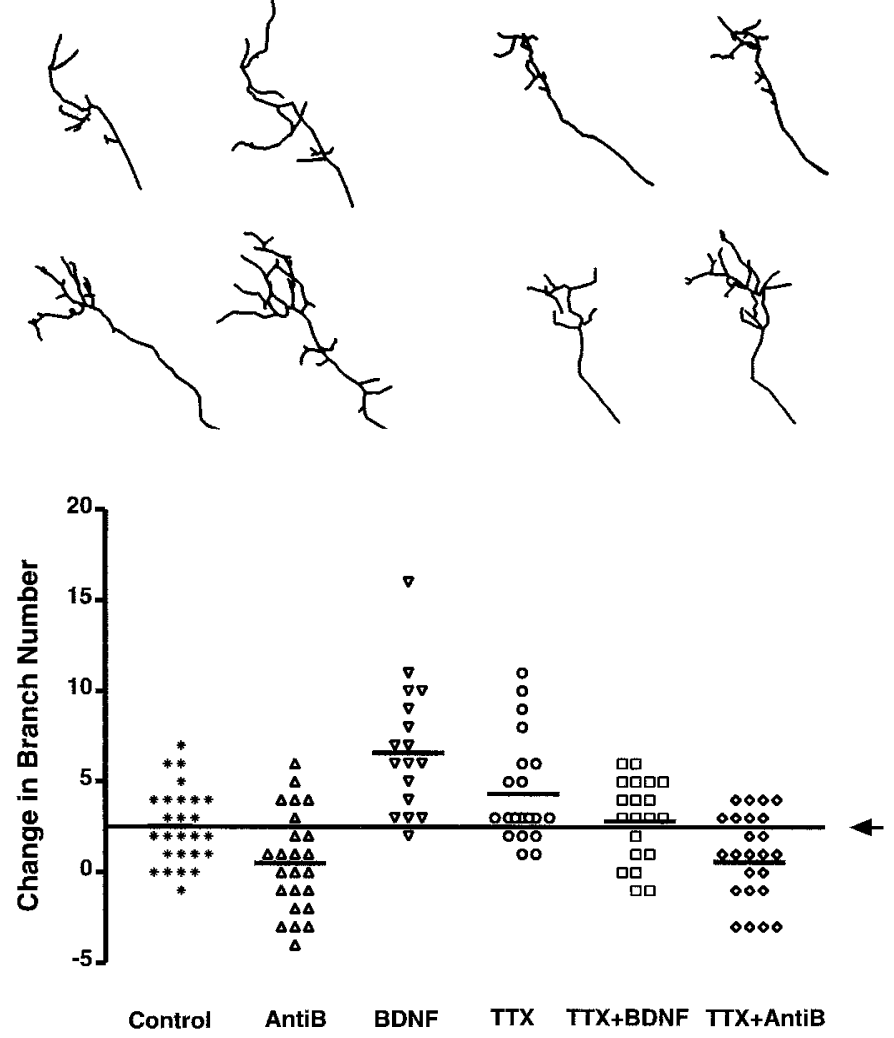

Figure 5. Variability in individual RGC arbor responses to activity blockade and tectal BDNF levels. The variability in responses in arbor morphologies for each treatment is illustrated for individual axons by the absolute difference in branch number between 0 and $24 \mathrm{hr}$ of treatment. For ease of comparison, the $x$-axis was shifted to coincide with the mean change in branch number for the control-treated axons $(2.56 \pm 0.41$ branches $/ 24 \mathrm{hr}$; arrow). Within the conditions, each symbol represents the change in branch number for an individual axon, and the horizontal bar indicates the mean change in branch number for that group. AntiB, Anti-BDNF.

eling was observed. More branches were added and eliminated in the absence of activity, but the rate at which individual preexisting branches grew remained the same. By $24 \mathrm{hr}$, this increased remodeling resulted in axon terminal arbors with increased complexity, because more branches were added than eliminated. Thus, the axon arbor morphologies resulting from activity blockade were not caused by interference with pruning mechanisms

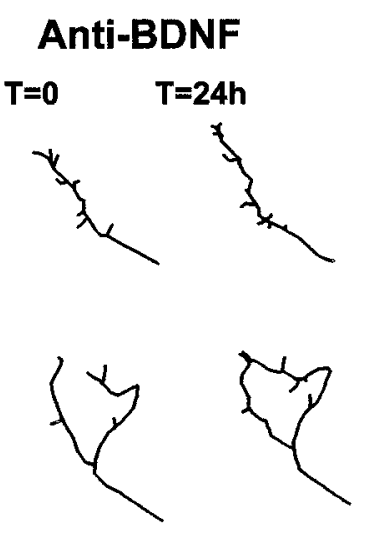

\section{TTX + Anti-BDNF}
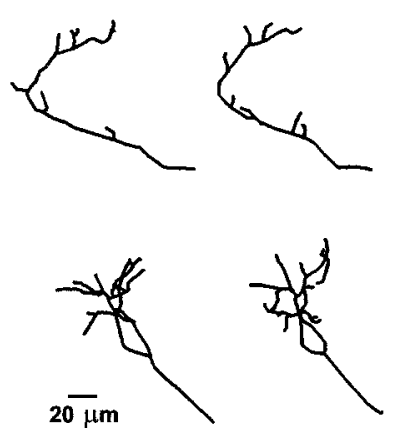

Figure 4. Xenopus RGC axon complexity is influenced by action potential activity and is modified by BDNF. Tracings of representative axonal arbors before and $24 \mathrm{hr}$ after control, TTX, BDNF, TTX + BDNF, antiBDNF, or TTX + anti-BDNF treatments illustrate the individual variability in arbor morphologies for each condition tested. During active arborization, RGC axons significantly increase their complexity as illustrated by the difference in their morphology 24 hr after initial observation (see controls). TTX-induced activity blockade and perturbations in tectal BDNF levels independently influence axon arbor complexity, but when combined, arbor complexities resembled that of controls.

but were rather a consequence of the increased remodeling that may result from interference with recognition mechanisms and/or synapse stabilization. A number of studies indicate that postsynaptic activity, mediated by the NMDA type of glutamate receptor (NMDA-R), is involved in the development and topographic refinement of RGC axon arbors (Cline and Constantine-Paton, 1990; Constantine-Paton et al., 1990; O'Rourke et al., 1994; Shatz, 1996; Rajan et al., 1999). Studies that have analyzed the effects of NMDA-R activity on axon arbor dynamics show that the dynamic remodeling of predominantly short branches $(<5$ $\mu \mathrm{m}$; termed spikes in the current studies) is increased by NMDA-R activity blockade (Rajan et al., 1999). However, in contrast to the effects of TTX, alterations in NMDA-R signaling has no net effect on overall axon branch number or morphology at $24 \mathrm{hr}$ (O'Rourke et al., 1994; Rajan et al., 1999). Thus, these and the present results support a role for neuronal activity in the stabilization of axonal branches and further suggest that both pre- and postsynaptic mechanisms are involved in axon branch stabilization.

The neurotrophins are attractive candidate signals for mediating activity-dependent synaptic remodeling during development. Neurotrophins are expressed and released in an activitydependent manner (Herzog et al., 1994; Lindholm et al., 1994; Thoenen, 1995; Blochl and Thoenen, 1996), can modulate synaptic activity both in vitro (Lohof et al., 1993; Kang and Schuman, 1995; Schuman, 1999) and in vivo (Prakash et al., 1996), can alter the activity-dependent segregation of inputs during development (Maffei et al., 1992; Cabelli et al., 1995), and are potent modulators of both axon (Cohen-Cory and Fraser, 1995) and dendritic (Cohen-Cory et al., 1991; McAllister et al., 1995) morphology. Collectively, this evidence has raised the intriguing possibility 


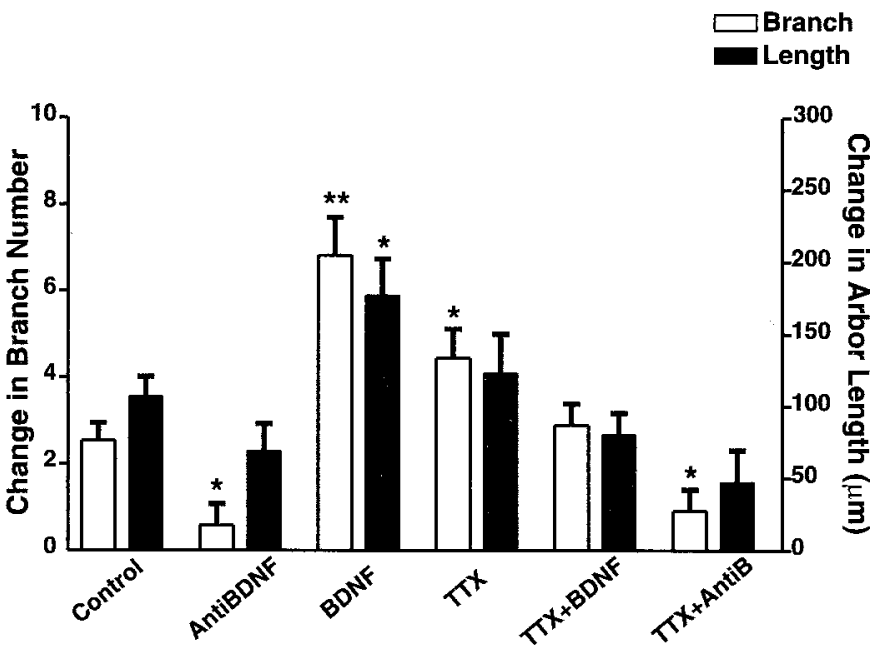

Figure 6. Action potential activity and tectal BDNF levels significantly influence the morphology of RGC axonal arbors at $24 \mathrm{hr}$. The effects of TTX and BDNF on arbor morphology over $24 \mathrm{hr}$ were evaluated quantitatively by measuring total arbor length and branch number in tadpoles treated with TTX alone, BDNF alone, anti-BDNF alone (AntiB), TTX + BDNF combined, or TTX + anti-BDNF combined. Values are presented as the change in initial value $24 \mathrm{hr}$ after treatment. For each parameter and condition the mean \pm SEM is shown. Statistical analysis was by one-way ANOVA using multiple comparison post hoc Tukey tests. ** indicates significantly different from control, $p \leq 0.001$; * indicates significantly different from control, $p \leq 0.05$.

that neurotrophins can act as activity-dependent retrograde signals that modulate neuronal connectivity (Katz and Shatz, 1996; Snider and Lichtman, 1996; McAllister et al., 1999). The present study has specifically addressed this issue by studying the combined effects of both blocking retinal action potential activity and altering endogenous tectal BDNF levels on the dynamics of RGC axon arborization in vivo. The results indicate that BDNF and neuronal activity use distinct mechanisms to modulate axon elaboration and refinement. The effects of blocking retinal activity with TTX on axon arbor dynamics significantly differed from the effects of altering tectal BDNF levels. TTX increased both the addition and elimination of axonal branches, indicating reduced branch stability, whereas alterations in BDNF tectal levels specifically affected branch addition and elongation, parameters that reflect growth. By following the dynamics of axon branch elaboration in detail, it was possible to dissect out the types of interactions between activity and BDNF in this developmental process. That different mechanisms are in effect is supported by the evidence that the combined treatment of tadpoles with TTX + BDNF or TTX + anti-BDNF affected axon branch dynamics in a manner that differed from the effects of treatment with either agent alone. Tectal injection of BDNF counteracted the effects of TTX on axon branch addition and prevented its effects on branch elimination. Blockade of action potential activity with TTX did not alter the effects of altered BDNF tectal levels, as is the case for the lengthening of previously established branches and the addition of new spikes. Therefore, the observation that activity blockade does not alter the effects of increased tectal BDNF or of reduced $\mathrm{BDNF}$ signaling indicates that the effects of endogenous BDNF are not directly dependent on action potential activity. Moreover, the observation that BDNF can modify the effects of activity blockade by reducing TTX-induced branch elimination indicates that BDNF can itself modulate the neuron's response to altered activity levels. Thus, although activity and BDNF interact to modulate axon arborization and remodeling, this interaction cannot simply be explained by a direct activity-dependent control of neurotrophic function.

One intriguing observation obtained from these studies is that either independent TTX or BDNF treatments increased axon branch addition, but when applied simultaneously RGC axons added new branches to a value that closely resembled that of controls. This significant result can be explained if neuronal activity blockade and BDNF trigger different and potentially opposing signaling mechanisms to each increase branch addition, but the significance of the branches extended under each circumstance differs. For example, in the absence of activity the probability that rudimentary branches are extended may increase as a response to failure to stabilize synaptic contacts (for review, see Katz and Shatz, 1996). BDNF, on the other hand, may specifically reinforce the initiation and elaboration of only those branches that are capable of establishing successful synaptic connections (Snider and Lichtman, 1996). Future studies that examine the relationship between axon branch elaboration and synapse formation and stabilization will help resolve this issue.

Observations of the morphological consequence of altering neurotrophin and activity levels on central neurons developing in culture have led to the suggestion that neurons must be active to respond to neurotrophins (Cohen-Cory et al., 1991; McAllister et al., 1996). For example, inhibiting action potential activity blocks the dramatic increase in dendritic arborization of cortical pyramidal neurons in culture elicited by BDNF, although activity inhibition also enhanced dendritic growth (McAllister et al., 1996). These observations of the combined effects of activity blockade and neurotrophins on dendritic morphology resemble the observations presented in this study on the final complexity of axonal arbors. However, the present study provides further dynamic evidence that BDNF and neuronal activity interact in a more complex way to modulate neuronal form. The combined in vivo time-lapse analysis of axon arbor dynamics and complexity indicates that neurotrophins can exert their effects even in the absence of activity but that the specificity of the response to neurotrophins and activity depends on the convergence of these two developmental signals. That BDNF can modify the effects of activity inhibition on dynamic morphological parameters supports a role for this neurotrophin as a potent mediator of morphological plasticity. One mechanism by which neurotrophins may modulate morphological plasticity is by modulating synaptic efficacy (Lohof et al., 1993; Kang and Schuman, 1995; Wang et al., 1998; Schuman, 1999), as has been demonstrated recently by BDNF's ability to modulate synaptic strength even in the absence of action potential activity (Rutherford et al., 1998). Thus, BDNF modulation of activity-dependent axonal branching may involve direct activation of signaling pathways common to the control of synaptic efficacy (Boulanger and Poo, 1999), growth cone behavior (Song et al., 1997), and collateral sprouting (Gallo and Letourneau, 1999).

In conclusion, the present observations demonstrate that BDNF modulates, but does not directly mediate, activitydependent branching and remodeling of RGC axon arbors in vivo. Although the results of this work favor the hypothesis that neuronal activity and neurotrophins use distinct but interactive mechanisms to modulate morphological plasticity, it also is plausible that neurotrophin function depends on neuronal activity at other regulatory levels, such as the expression and release of neurotrophic factors. Indeed, BDNF expression in the Xenopus optic tectum is developmentally regulated (Cohen-Cory and 
Fraser, 1994), being maximal during the period of active RGC axon arborization and synapse formation [determined both electrophysiologically (Wu et al., 1996; Zhang et al., 1998) and by the localization of synaptic vesicle proteins to RGC axon terminals (Lom et al., 1998)]. Thus, by interacting at multiple levels, BDNF and neuronal activity may exert fine regulatory control of neuronal development and function, from the control of neuronal morphology to the formation and stabilization of individual synaptic contacts.

\section{REFERENCES}

Antonini A, Stryker MP (1993a) Rapid remodeling of axonal arbors in the visual cortex. Science 260:1819-1821.

Antonini A, Stryker MP (1993b) Development of individual geniculocortical arbors in cat striate cortex and effects of binocular impulse blockade. J Neurosci 13:3549-3573.

Blochl A, Thoenen H (1996) Localization of cellular storage compartments and sites of constitutive and activity-dependent release of nerve growth factor (NGF) in primary cultures of hippocampal neurons. Mol Cell Neurosci 7:173-190.

Boulanger L, Poo M-m (1999) Gating of BDNF-induced synaptic potentiation by cAMP. Science $284: 1982-1984$.

Cabelli RJ, Hohn A, Shatz CJ (1995) Inhibition of ocular dominance column formation by infusion of NT-4/5 or BDNF. Science 267:1662-1666.

Catalano SM, Shatz CJ (1998) Activity-dependent cortical target selection by thalamic axons. Science 281:559-562.

Cline HT (1991) Activity-dependent plasticity in the visual system of frogs and fish. Trends Neurosci 14:104-111.

Cline HT, Constantine-Paton M (1990) NMDA receptor agonists and antagonists alter retinal ganglion cell arbor structure in the developing frog retinotectal projection. J Neurosci 10:1197-1216.

Cohen-Cory S, Fraser SE (1994) BDNF in the development of the visual system of Xenopus. Neuron 12:747-761.

Cohen-Cory S, Fraser SE (1995) Effects of brain-derived neurotrophic factor on optic axon branching and remodeling in vivo. Nature 378:192-196

Cohen-Cory S, Dreyfus CF, Black IB (1991) NGF and excitatory neurotransmitters regulate survival and morphogenesis of cultured cerebellar Purkinje cells. J Neurosci 11:462-471.

Cohen-Cory S, Escandón E, Fraser SE (1996) The cellular patterns of BDNF and trkB expression suggest multiple roles for BDNF during Xenopus visual system development. Dev Biol 179:102-115.

Constantine-Paton M, Cline HT, Debski E (1990) Patterned activity, synaptic convergence, and the NMDA receptor in developing visual pathways. Annu Rev Neurosci 13:129-154.

Crair MC (1999) Neuronal activity during development: permissive or instructive? Curr Opin Neurobiol 9:88-93.

Dantzker JL, Callaway EM (1998) The development of local, layerspecific visual cortical axons in the absence of extrinsic influences and intrinsic activity. J Neurosci 18:4145-4154.

Gallo G, Letourneau PC (1999) A balance of signals sets axons on the right track. Curr Biol 9:R490-R492.

Ghosh A, Carnahan J, Greenberg ME (1994) Requirement for BDNF in activity-dependent survival of cortical neurons. Science 263:1618-1623.

Herzog KH, Bailey K, Barde YA (1994) Expression of the BDNF gene in the developing visual system of the chick. Development 1206:1643-1649.

Kang H, Schuman EM (1995) Long lasting neurotrophin-induced enhancement of synaptic transmission in the adult hippocampus. Science 267:1658-1662.

Katz LC, Shatz CJ (1996) Synaptic activity and the construction of cortical circuits. Science 274:1133-1138.

Kobayashi T, Nakamura H, Yasuda M (1990) Disturbance of refinement of retinotectal projection in chick embryos by tetrodotoxin and grayanotoxin. Dev Brain Res 57:29-35.
Lindholm D, Castren E, Berzaghi M, Blochl A, Thoenen H (1994) Activity-dependent and hormonal regulation of neurotrophin mRNA levels in the brain-implications for neuronal plasticity. J Neurobiol 25:1362-1372.

Lohof AM, Ip NY, Poo M-M (1993) Potentiation of developing neuromuscular synapses by the neurotrophins NT-3 and BDNF. Science: 363:350-353.

Lom B, Nguyen L, Prado M, Cohen-Cory S (1998) Expression of synaptic proteins in the developing Xenopus laevis visual system. Soc Neurosci Abstr 24:2000.

Maffei L, Berardi N, Domenici L, Parisi V, Pizzoruso T (1992) Nerve growth factor (NGF) prevents the shift in ocular dominance distribution of visual cortical neurons in monocularly deprived rats. J Neurosci 12:4651-4662.

McAllister AK, Lo DC, Katz LC (1995) Neurotrophins regulate dendritic growth in developing visual cortex. Neuron 15:791-803.

McAllister AK, Katz LC, Lo DC (1996) Neurotrophin regulation of cortical dendritic growth requires activity. Neuron 17:1057-1064.

McAllister AK, Katz LC, Lo DC (1999) Neurotrophins and synaptic plasticity. Annu Rev Neurosci 22:295-318.

Meyer RL (1983) Tetrodotoxin inhibits the formation of refined retinotopography in goldfish. Dev Brain Res 6:293-298.

Nakamura H, O'Leary DD (1989) Inaccuracies in initial growth and arborization of chick retinotectal axons followed by course corrections and axon remodeling to develop topographic order. J Neurosci 9:3776-3795.

Nieuwkoop PD, Faber J (1956) Normal table of Xenopus laevis. Amsterdam: Elsiever North Holland.

O'Rourke NA, Fraser SE (1990) Dynamic changes in optic fiber terminal arbors lead to retinotopic map formation: an in vivo confocal microscopic study. Neuron 5:159-171.

O'Rourke NA, Cline HT, Fraser SE (1994) Rapid remodeling of retinal arbors in the tectum with and without blockade of synaptic transmission. Neuron 12:921-934.

Prakash N, Cohen-Cory S, Frostig RD (1996) Rapid and opposite effects of BDNF and NGF on the functional organization of the adult cortex in vivo. Nature 381:702-706.

Rajan I, Witte S, Cline HT (1999) NMDA receptor activity stabilizes presynaptic retinotectal axons and postsynaptic optic tectal cell dendrites in vivo. J Neurobiol 38:357-368.

Reh TA, Constantine-Paton M (1985) Eye specific segregation requires neural activity in three-eyed Rana pipiens. J Neurosci 5:1132-1143.

Roskies A, Friedman GC, O'Leary DD (1995) Mechanisms and molecules controlling the development of retinal maps. Perspect Dev Neurobiol 3:63-75.

Rutherford LC, Nelson SB, Turrigiano GG (1998) BDNF has opposite effects on the quantal amplitude of pyramidal neuron and interneuron excitatory synapses. Neuron 21:521-530.

Schuman EM (1999) Neurotrophin regulation of synaptic transmission. Curr Opin Neurobiol 9:105-109.

Shatz CJ (1996) Emergence of order in visual system development. Proc Natl Acad Sci USA 93:602-608.

Snider WD, Lichtman JW (1996) Are neurotrophins synaptotrophins? Mol Cell Neurosci 7:433-442.

Song HJ, Ming GL, Poo MM (1997) cAMP-induced switching in turning direction of nerve growth cones. Nature 388:275-279.

Sretavan DW, Shatz CJ, Stryker MP (1988) Modification of retinal ganglion cell axon morphology by prenatal infusion of tetrodotoxin. Nature 336:468-471.

Thoenen H (1995) Neurotrophins and neural plasticity. Science 270:593-598.

Wang XH, Berninger B, Poo M-m (1998) Localized synaptic actions of neurotrophin-4. J Neurosci 18:4985-4992.

Wu G, Malinow R, Cline HT (1996) Maturation of a central glutamatergic synapse. Science 274:972-976.

Zhang LI, Tao HW, Holt CE, Harris WA, Poo M-m (1998) A critical window for cooperation and competition among developing retinotectal synapses. Nature 395:37-44. 